\title{
Gender Differences in Age-Related Changes in Left and Right Ventricular Geometries and Functions
}

\author{
- Echocardiography of a Healthy Subject Group -
}

\author{
Masao Daimon, MD; Hiroyuki Watanabe, MD; Yukio Abe, MD; Kumiko Hirata, MD; \\ Takeshi Hozumi, MD; Katsuhisa Ishii, MD; Hiroshi Ito, MD; Katsuomi Iwakura, MD; \\ Chisato Izumi, MD; Masunori Matsuzaki, MD; Shinichi Minagoe, MD; Haruhiko Abe, MD; \\ Kazuya Murata, MD; Satoshi Nakatani, MD; Kazuaki Negishi, MD; Ken Yoshida, MD; \\ Kazuaki Tanabe, MD; Nobuhiro Tanaka, MD; Kotaro Tokai, MD; \\ Junichi Yoshikawa, MD for the Japanese Normal Values \\ for Echocardiographic Measurements Project (JAMP) Study Investigators
}

\begin{abstract}
Background: The purpose of the present study was to investigate gender differences in age-related changes of left ventricular (LV) and right ventricular (RV) geometries and functions throughout the entire adult age range using the Japanese Normal Values for Echocardiographic Measurements Project (JAMP) study database.
\end{abstract}

\begin{abstract}
Methods and Results: Seven hundred healthy volunteers (aged 20-79 years) underwent 2-dimensional and Doppler echocardiography. The subjects were stratified into 6 different age groups and then stratified by gender in each age group. LV diastolic function was assessed from pulsed wave Doppler measurements of mitral early (E) and late (A) inflow velocities and tissue Doppler measurements of mitral early (e') and late (a') annular velocities. LV volume decreased and LV mass increased with age to a similar extent in both men and women. Furthermore, for subjects $<50$ years, women had significantly greater $\mathrm{E}, \mathrm{E} / \mathrm{A}$ ratio and e' than men, but these parameters were similar between genders in subjects $>50$ years. In addition, there was a significant interaction between age and gender that affected the differences in $E$, e' and $E / e^{\prime}$ among the groups $(P<0.03, P<0.01$, and $P<0.03$, respectively; ANOVA). There were no gender differences in age-related changes in RV parameters.
\end{abstract}

Conclusions: Gender differences were found in age-related changes in LV diastolic function in a healthy population. Gender differences should be considered for optimal diagnosis and management of cardiovascular disease. (Circ J 2011; 75: 2840-2846)

Key Words: Echocardiography; Gender; Ventricular function

$\mathbf{G}$ ender-based differences in the management and outcomes of cardiovascular disease have been studied extensively. ${ }^{1-4}$ In women, heart failure (HF) is associated more with left ventricular (LV) diastolic dysfunction than in men, whereas LV systolic dysfunction is the predominant cause of HF in men. ${ }^{5-8}$ In particular, elderly women fre- quently have HF associated with a normal LV ejection fraction. ${ }^{2}$ Recent studies have also emphasized the importance of gender differences in the management of cardiovascular disease..$^{1,8,9}$ Thus, recognition of gender difference in LV geometry and function has important implications for the optimal diagnosis and management of cardiovascular disease.

Received April 8, 2011; revised manuscript received August 17, 2011; accepted August 18, 2011; released online September 23, 2011 Time for primary review: 24 days

Juntendo University, Tokyo (M.D.); Sakakibara Heart Institute, Tokyo (H.W.); Osaka City General Hospital, Osaka (Y.A.); Higashisumiyoshi Morimoto Hospital, Osaka (K.H.); Osaka City University, Osaka (T.H.); Kansai Electric Power Hospital, Osaka (K. Ishii); Sakurabashi Watanabe Hospital, Osaka (H.I., K. Iwakura); Tenri Hospital, Tenri (C.I.); Yamaguchi University, Ube (M.M., K.M.); National Hospital Organization Kagoshima Medical Center, Kagoshima (S.M.); National Hospital Organization Osaka Minami Medical Center, Osaka (H.A.); National Cerebral and Cardiovascular Center, Suita (S.N.); Gunma University, Maebashi (K.N.); Osaka Ekisaikai Hospital, Osaka (K.Y.); Kobe City Medical Center General Hospital, Kobe (K. Tanabe); Tokyo Medical University, Tokyo (N.T.); Nihon University, Tokyo (K. Tokai); and Nishinomiya Watanabe Cardiovascular Center Hospital, Nishinomiya (J.Y.), Japan

Present address: Wakayama Medical University, Wakayama (K.H.); Okayama University, Okayama (H.I.); Osaka University, Osaka (S.N.); and Shimane University, Izumo (K. Tanabe), Japan

Mailing address: Junichi Yoshikawa, MD, Nishinomiya Watanabe Cardiovascular Center Hospital, 3-25 Ikeda-cho, Nishinomiya 662-0911, Japan. E-mail: yoshikawaj@n-watanabe-hosp.jp

ISSN-1346-9843 doi:10.1253/circj.CJ-11-0364

All rights are reserved to the Japanese Circulation Society. For permissions, please e-mail: cj@j-circ.or.jp 
Echocardiography is now recognized as an integral diagnostic tool that provides non-invasive quantification of cardiac chamber size, ventricular mass and function in the clinical setting. Furthermore, technological advancements in Doppler echocardiography have permitted quantitative assessment of ventricular diastolic as well as systolic function. Thus, echocardiography has become an important cardiac imaging technique to evaluate the efficacy of drug treatments or new therapeutic strategies in many clinical trials. Previous investigations reported age-related changes in LV diastolic indices derived from Doppler echocardiography. ${ }^{10-13}$ There is little information, however, on the effect of gender on age-related changes in LV geometry and function throughout the entire adult age range in healthy subjects. Furthermore, the age-related changes in right ventricular (RV) geometry and function have also not been well investigated previously.

Recently, we conducted a multicenter study, the Japanese Normal Values for Echocardiographic Measurements Project (JAMP) ${ }^{14}$ to determine normal values for echocardiographic measurements throughout the entire adult age range in a large, healthy population. To our knowledge, the JAMP study is the only study in which echocardiographic parameters were measured in a large population of Asian healthy volunteers. Therefore, the purpose of the present study was to investigate gender differences in age-related changes in LV and RV geometries and functions throughout the entire adult age range using the JAMP study database.

\section{Methods}

\section{Subjects}

A total of 700 healthy volunteers, aged 20-79 years, were registered in the JAMP study at 17 collaborating institutions (Table). A standard protocol for echocardiographic measurements was established for the present study. Subjects were excluded based on the following criteria: any history of hyperlipidemia, hypertension, diabetes mellitus, renal failure, cardiovascular disease, abnormal electrocardiographic findings including cardiac arrhythmia and bundle branch block, or abnormal echocardiographic findings (LV wall motion abnormalities or significant valvular disease). Subjects were also excluded if they had poor echocardiographic images, significant fever, anemia or hypertension (systolic $\geq 135 \mathrm{mmHg}$ and/or diastolic $\geq 85 \mathrm{mmHg}$ ) at the time of echocardiography or if they were under any influence that could affect the echocardiographic measurements. The study protocol was approved by the local hospital ethics committees, and informed consent was obtained from all subjects.

\section{Echocardiographic Chamber Quantification and Doppler Echocardiography}

The methods and results of chamber quantification and Doppler Echocardiography in the JAMP study have been described previously. ${ }^{14}$ In brief, echocardiography was performed using commercially available equipment with tissue Doppler capabilities at each institution according to a standard protocol. Two-dimensional (2-D) and color Doppler imaging were performed to screen for significant valvular disease. In each subject, cardiac chamber quantification on 2-D echocardiography was performed according to guidelines provided by the American Society of Echocardiography. ${ }^{15} \mathrm{LV}$ end-diastolic volume (EDV) and end-systolic volume (ESV) were measured using the biplane Simpson disk method. LV mass was calculated based on the area-length formula as previously described. ${ }^{15}$ Maximum left atrial (LA) and LV volumes were

\begin{tabular}{|lcc|}
\hline \multicolumn{2}{c}{ Table. Subject Characteristics } \\
\cline { 2 - 3 } & \multicolumn{2}{c|}{ Total } \\
\cline { 2 - 3 } Male $(\mathbf{n}=\mathbf{3 8 3})$ & Female $(\mathbf{n}=\mathbf{3 1 7})$ \\
Height $(\mathrm{cm})$ & $44 \pm 14$ & $44 \pm 14$ \\
Weight $(\mathrm{kg})$ & $170 \pm 6$ & $157 \pm 6$ \\
Body surface area $\left(\mathrm{m}^{2}\right)$ & $65 \pm 9$ & $51 \pm 7$ \\
SBP $(\mathrm{mmHg})$ & $1.7 \pm 0.1$ & $1.5 \pm 0.1$ \\
DBP $(\mathrm{mmHg})$ & $119 \pm 11$ & $114 \pm 12$ \\
Heart rate $($ beats/min) & $73 \pm 8$ & $70 \pm 9$ \\
Age group $(\mathrm{n})$ & $64 \pm 9$ & $64 \pm 9$ \\
20-29 years & & \\
$30-39$ years & 84 & 63 \\
$40-49$ years & 79 & 79 \\
$50-59$ years & 88 & 66 \\
$60-69$ years & 72 & 60 \\
$70-79$ years & 40 & 35 \\
\end{tabular}

SBP, systolic blood pressure; DBP, diastolic blood pressure.

measured using the biplane Simpson disk method using 2-D images from the apical 4- and 2-chamber views. Assessment of RV size was done by measuring RV end-diastolic area and end-systolic area from the apical 4-chamber view. For assessing RV function, RV fractional area change ${ }^{15}$ was calculated using the equation $100 \times($ end-diastolic area-end-systolic area)/ end-systolic area. Each parameter obtained from chamber quantification was indexed for body surface area when appropriate.

Pulsed wave Doppler imaging of mitral inflow and tissue Doppler imaging (TDI) of mitral annular motion at the septum were also performed to assess LV diastolic function as previously described. ${ }^{16}$ Peak velocities of early (E) and late (A) diastolic flow, E/A ratio, and early flow deceleration time (Dct) were measured from pulsed wave Doppler imaging of mitral inflow. Early (e') and late (a') diastolic annular velocities were measured from TDI. The ratio of mitral E to TDI e' was also calculated using these measurements.

\section{Statistical Analysis}

Data analysis was performed using SPSS 17.0 (SPSS, Chicago, IL, USA). Subjects were stratified into 6 different age groups and then further stratified by gender. All results are expressed as mean $\pm \mathrm{SD}$. Univariate regression analysis was used to assess liner correlations between age and the echocardiographic parameters. Unpaired t-test was used to compare the echocardiographic parameters between men and women in the same age group. Two-way analysis of variance (ANOVA) was used to determine if there was an interaction effect between gender and age that influenced any observed differences in the echocardiographic parameters among the groups. If there was a significant interaction effect between them, we considered that gender difference was significant in the age-related change in the echocardiographic parameter. $\mathrm{P}<0.05$ was considered statistically significant.

\section{Results}

\section{Volumes, Ejection Fraction and Mass}

Both LV EDV and ESV indexes decreased with age to a similar extent in men and women, but these indices were significantly greater in men than in women in 3 age groups (2029 years, 30-39 years and 50-59 years; Figures 1A,B). In 

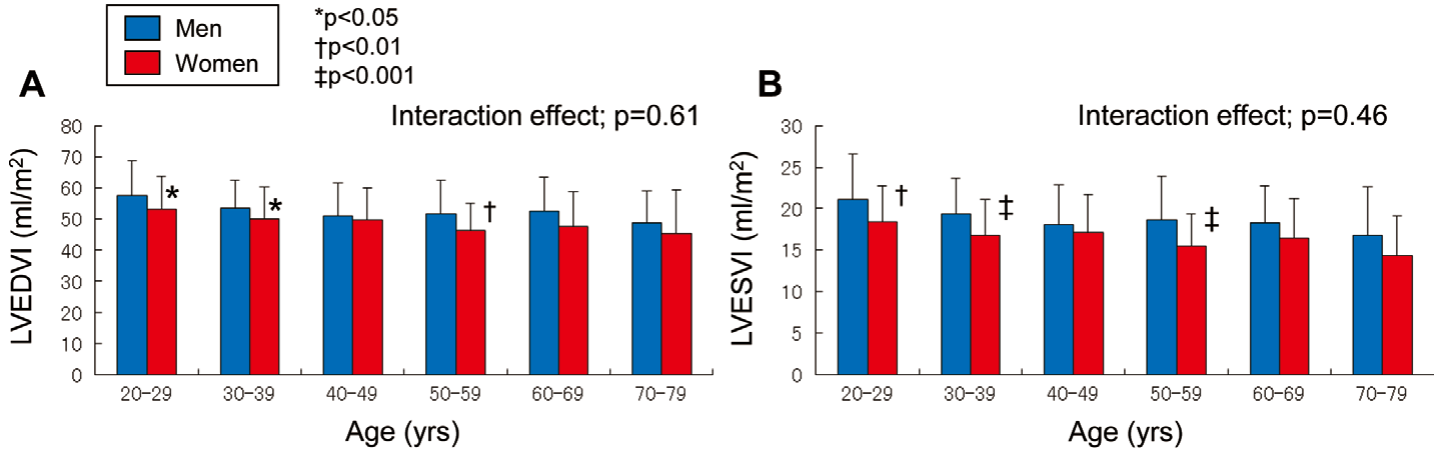

C

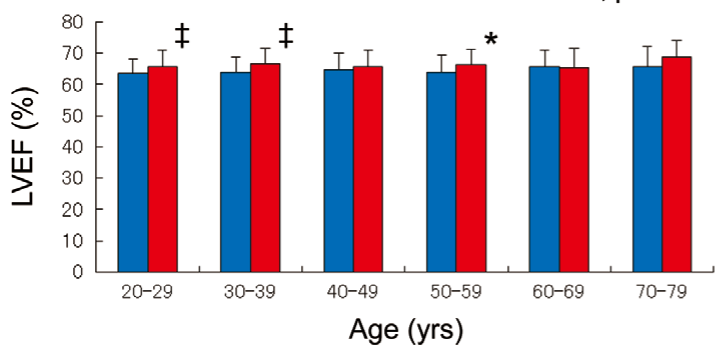

Figure 1. Effects of age and gender on left ventricular (LV) volumes and ejection fraction. Among subjects aged 20-29 years, 30-39 years and 50-59 years, (A) LV end-diastolic volume index (LVEDVI) and (B) LV end-systolic volume index (LVESVI) were greater, and (C) LV ejection fraction (LVEF) was lower in men than in women. There were no significant interaction effects, however, between age and gender that affected the differences in these parameters among the groups. ${ }^{*} P<0.05,{ }^{t} P<0.01,{ }^{\ddagger} P<0.001$.

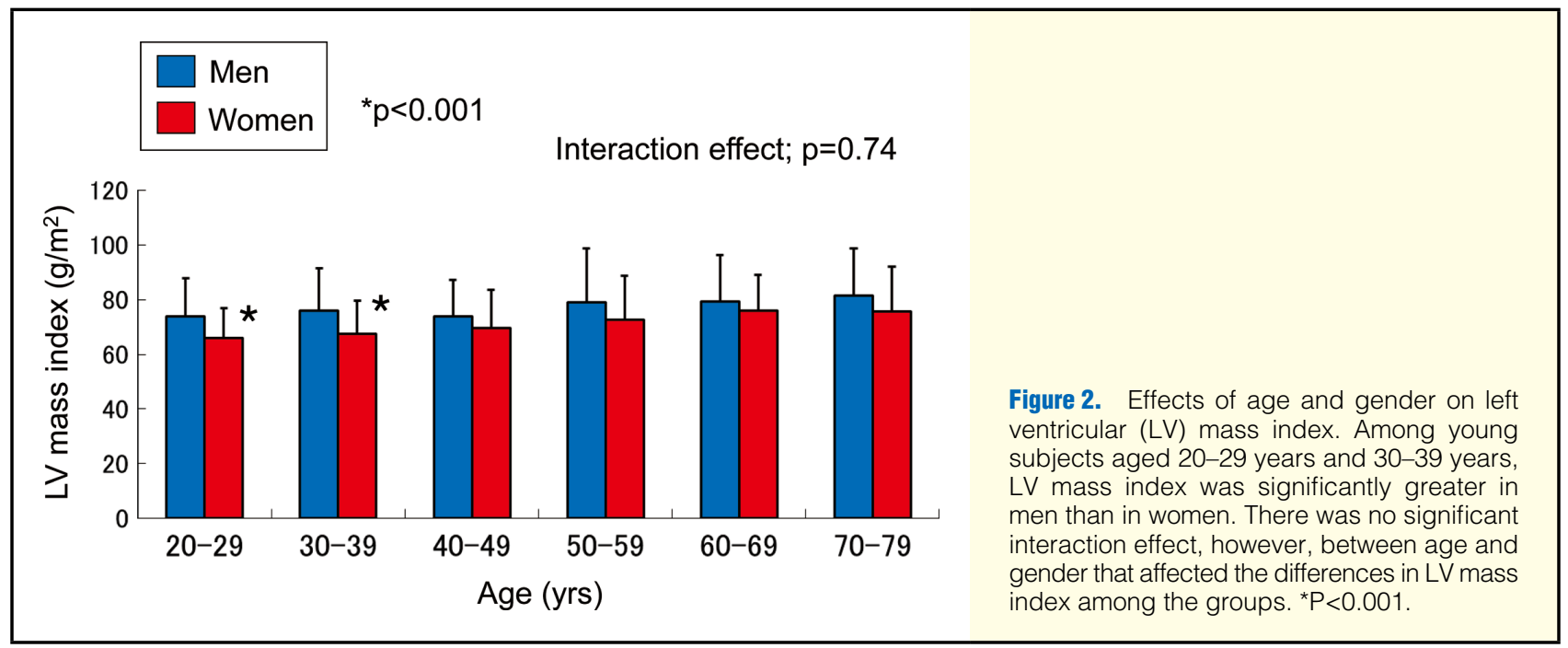

addition, LV ejection fraction was lower in men than in women in the same 3 age groups (Figure $1 \mathrm{C}$ ). There were no significant interaction effects, however, between gender and age that influenced the observed differences in the LV volume indices and ejection fraction. LV mass index increased with age to a similar extent in both men and women (Figure 2). LV mass index was significantly lower in women than in men in the 2 youngest age groups (20-29 years and 30-39 years), and the increase in LV mass index with age seemed more pronounced in women than men. There was no significant interaction effect, however, between age and gender that affected the differences in LV mass index among the groups.

\section{Diastolic Parameters and LA Volume}

The results of mitral inflow and TDI are shown in Figures 3,4, respectively. A decline in mitral peak $\mathrm{E}$ velocity, an increase in mitral peak A velocity, a decline in mitral E/A ratio and an increase in mitral Dct were observed with age in both men and women. Of note, mitral peak E velocity and E/A ratio were significantly greater in women than men in subjects $<50$ years but were similar between men and women in subjects $\geq 50$ years. In addition, there was a significant interaction effect 

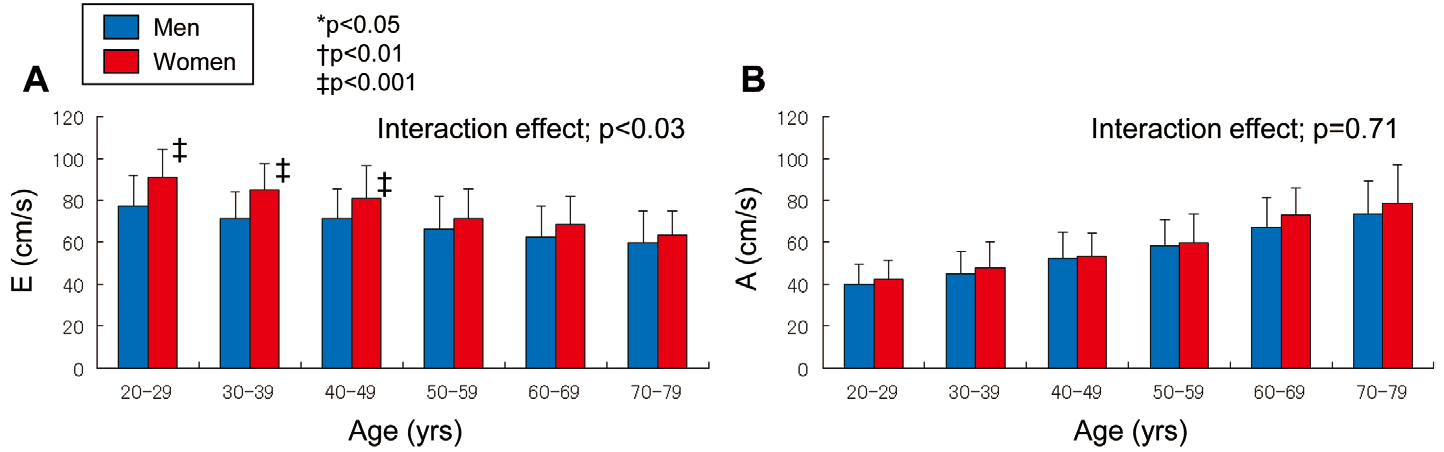

C

D
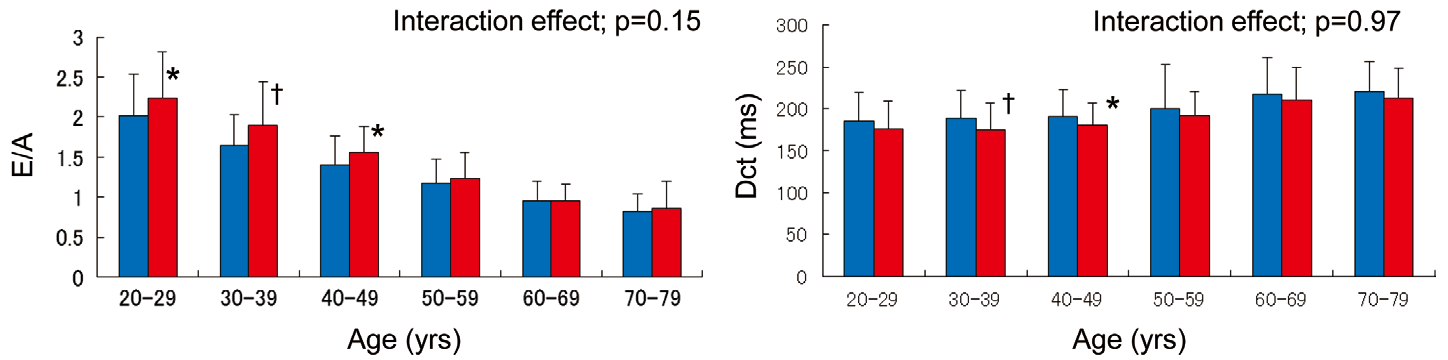

Figure 3. Effects of age and gender on mitral inflow parameters. Among subjects aged 20-29 years, 30-39 years and 40-49 years, (A) mitral peak E velocity and (C) mitral E/A ratio were greater in women than in men, and (D) among subjects aged 30-39 years and 40-49 years, mitral early flow deceleration time (Dct) was shorter in women than in men. There was a significant interaction effect between gender and age that affected the differences in peak $E$ wave velocity among the groups. ${ }^{*} P<0.05,{ }^{t} P<0.01$, $\neq \mathrm{P}<0.001$ (ANOVA).
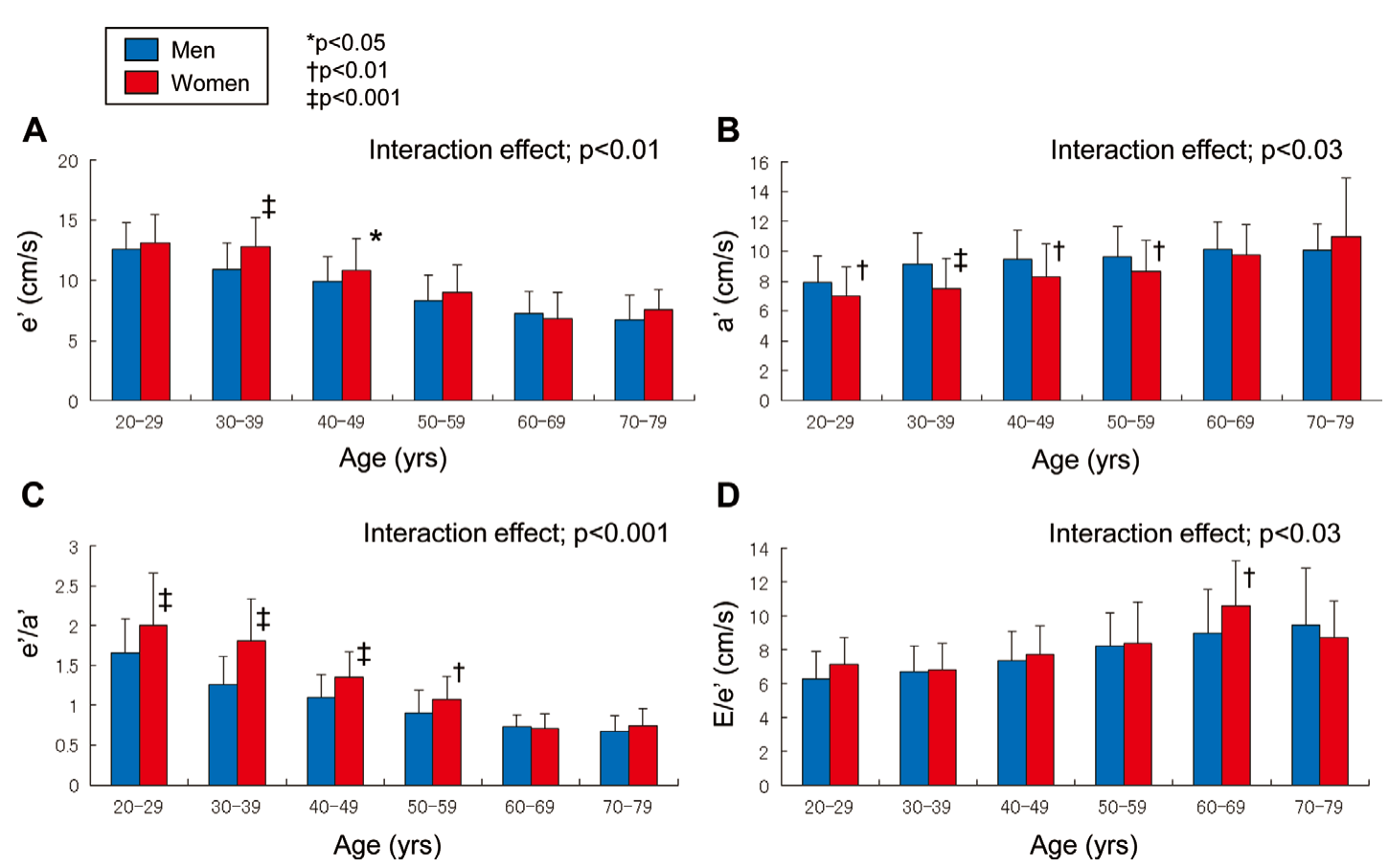

D

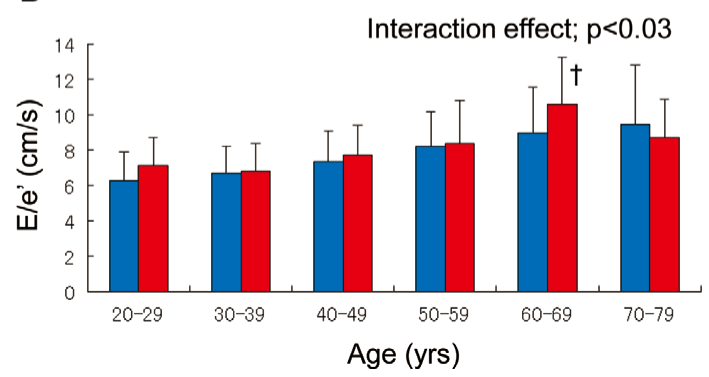

Figure 4. Effects of age and gender on tissue Doppler imaging (TDI) parameters. Among subjects aged 30-39 years and 4049 years, (A) TDI e' velocity was greater in women than in men. Among subjects aged 20-29 years, 30-39 years, 40-49 years and 50-59 years, (B) TDI a' velocity was lower in women than in men, and (C) TDI e'/a' ratio was greater in women than in men. Among subjects aged 60-69 years, (D) E/e' ratio was greater in women than in men. There were significant interaction effects between gender and age that affected the differences in all of these parameters among the groups. ${ }^{*} P<0.05,+P<0.01, \neq P<0.001$ (ANOVA). 

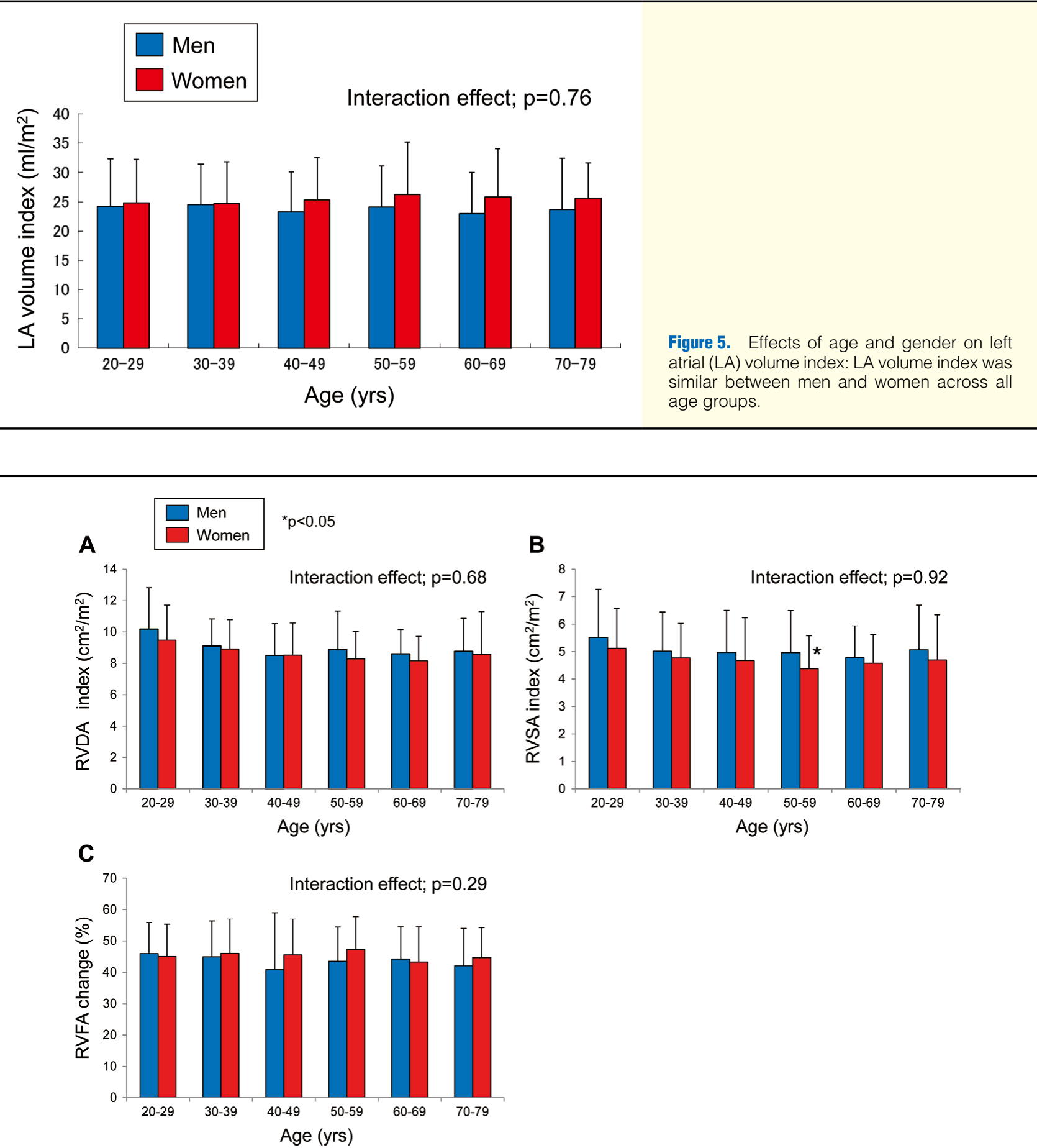

Figure 6. Effects of age and gender on right ventricular (RV) area and fractional area change. Both diastolic and systolic RV area indices slightly decreased with age similarly in both men and women. RV fractional area change was similar in both men and women across all age groups. RVDA, RV diastolic area; RVFA, RV fractional area; RVSA, RV systolic area. *P<0.05.

$(\mathrm{P}<0.03$; ANOVA) between gender and age that affected the differences in peak $\mathrm{E}$ wave velocity among the groups. As shown in Figure 4, younger women had higher TDI, e' velocity and e'/a' ratio but lower a' than younger men; but there were no differences in these parameters between older men and women. Moreover, ANOVA indicated a significant interaction effect between gender and age that influenced the differences in all of the TDI-derived parameters among the groups.

For age-related changes in LA volume, women tended to have a larger age-related increase in LA volume than men (Figure 5). There was no significant interaction effect, however, between gender and age that influenced the differences in LA volume. 


\section{RV Size and Systolic Function}

The results of RV area and fractional change are given in Figure 6. There were no significant differences in RV diastolic and systolic area indices, or in RV area fractional change between men and women, except for RV systolic area index among subjects aged 50-59 years. Accordingly, there were no gender differences in age-related changes in these RV parameters.

\section{Discussion}

In the present study we found significant gender differences in the age-related changes in LV diastolic parameters assessed on mitral inflow and TDI. Overall, younger women had better LV diastolic parameters and lower LV mass index compared with younger men, whereas these parameters were similar in older ( $>50$ years) men and women. In contrast, there were no gender differences in age-related changes in RV size and systolic function. The present results indicate that gender differences should be considered in association with age in the management of cardiovascular disease. In addition, recognition of these differences is critical not only in routine clinical practice, but also in interpreting the results of clinical trials that use echocardiography to measure cardiac geometry and function.

Gender-based differences in the management and outcomes of patients with cardiovascular disease have been widely recognized and extensively investigated. ${ }^{1-4}$ Recently, Okura et al reported gender-specific changes in LV relaxation with age in healthy individuals without arrhythmias, abnormal echocardiographic findings, a history of heart disease or hypertension. ${ }^{17}$ In that study, subclinical conditions such as diabetes mellitus or renal failure that might have affected LV diastolic parameters could not be completely excluded. In contrast, we enrolled healthy volunteers without any systemic conditions including diabetes or renal failure that might have altered LV diastolic parameters in an attempt to assess only normal echocardiographic parameters. In fact, LV diastolic parameters in the Okura et al study appear to be attenuated compared with the same parameters measured in the same age group in the present subjects. Munagala et al and Redfield et al similarly reported age-related changes in LV diastolic parameters, ${ }^{10,11}$ but because their subjects consisted of people aged $>45$ years, they were not able to identify gender-specific differences in echocardiographic LV parameters over the entire adult age range.

The effect of estrogen and postmenopausal status on smooth muscle proliferation ${ }^{18}$ and vascular function ${ }^{19,20}$ may play a role in gender-based differences in echocardiographic LV parameters. In the present study LV diastolic parameters were relatively worse in women than in men over 50 , whereas younger women had better diastolic parameters than men in the same age range. Menopause usually occurs at the age of approximately 50, and rapid changes in LV diastolic parameters in women over 50 seem to be consistent with postmenopausal status.

Although we found gender differences in the changes in LV diastolic parameters with age, the present data did not suggest deteriorated LV diastolic function in elderly women compared with elderly men. At least, no significant differences in LV diastolic parameters were found between elderly men and women. The majority of diastolic HF occurs in association with hypertension ${ }^{21,22}$ or diabetes mellitus. ${ }^{23,24}$ Thus, future studies need to explore the mechanisms responsible for hypertension- or diabetes-induced diastolic dysfunction in elderly women. Moreover, the present study suggests that gender differences in age-related changes in LV geometry and function should be considered in the investigation of the pathophysiology of both diastolic and systolic HF.

\section{Study Limitations}

We investigated LV geometry and function in totally healthy volunteers in the present study. Therefore, the number of subjects aged 70-79 years was small, because of the difficulty in finding healthy volunteers in that age range without any conditions that affect echocardiographic parameters. Furthermore, the medical histories of participants were reviewed at each institution, therefore, the presence of unrecognized cardiovascular disease cannot be ruled out. In addition, echocardiographic measurements were performed at each institution according to a standard protocol. Thus, inter-observer variability might have affected the echocardiographic measurements. The standard deviations of the measurements, however, were small and similar to those reported in previous studies, $, 9,11$ and the influence of inter-observer variability was considered to be negligible.

We excluded subjects with a history of hypertension and those with high blood pressure at the time of echocardiography, therefore we did not take into consideration the influence of blood pressure on LV geometry in the current study. There was, however, a slight age-related increase in blood pressure both in men and women, as presented in our previous study. ${ }^{14}$ This age-related change of blood pressure might have influenced the present results.

\section{Conclusions}

We identified gender differences in age-related changes in LV geometry and function in a healthy population. The present results indicate the need for consideration of these gender differences in the optimal diagnosis and management of cardiovascular disease.

\section{Acknowledgments}

We thank many colleagues for their assistance in the echocardiography recording and measurements: Sakiko Miyazaki, MD; Hiroyuki Tsurumi, MT (Juntendo University); Kiyomi Saito, MT (Sakakibara Heart Institute); Akira Motobayashi, MT; Noriyo Fujii, MT; Rieko Nakamura, MT (Osaka City General Hospital); Ryoichi Kita, MT; Yukari Kadotani, MT; Reiko Miyahana, MT (Higashisumiyoshi Morimoto Hospital); Shota Fukuda, MD; Kazuya Fujioka, MT (Osaka City University); Chizu Miyasaka, MT; Kyoko Teramura, MT (Kansai Electric Power Hospital); Masakazu Ueda, MT; Kayo Komura, MT (Sakurabashi Watanabe Hospital); Shuichi Takahashi, MT; Sumiyo Hashiwada, MT; Kazuyo Kuwano, MT (Tenri Hospital); Yasuaki Wada, MT; Yoshio Nose, MD; Takeo Tanaka MD (Yamaguchi University); Masao Okuno, MT (National Hospital Organization Kagoshima Medical Center); Keiko Katsuki, MT; Seiko Umekawa, MT; Norio Tanaka, MT (National Hospital Organization Osaka Minami Medical Center); Jun Tanaka, MD; Makoto Amaki, MD; Yumiko Suda, MT (National Cerebral and Cardiovascular Center); Tomoko Takada, MT; Sakiko Inokuma, MT; Rieko Takahashi, MD (Gunma University); Toshihiro Kawasaki, MT; Kumiko Maeda, MT; Hiroe Sunada, MT (Osaka Ekisaikai Hospital); Kazuto Yamaguchi, MT (Kobe City Medical Center General Hospital); Tomoko Shoda, MD; Nori Takahashi, MT; Naho Aikawa, MD (Tokyo Medical University); Rieko Seki, MT; Sachiko Kawaguchi, MT (Nihon University).

\section{Disclosure}

None of the authors have relationships to disclose.

\section{References}

1. Rumsfeld JS, Masoudi FA. Sex differences: Implications for heart failure care. Eur Heart J 2004; 25: 101-103. 
2. Regitz-Zagrosek V, Oertelt-Prigione S, Seeland U, Hetzer R. Sex and gender differences in myocardial hypertrophy and heart failure. Circ J 2010; 74: 1265-1273.

3. Arain FA, Kuniyoshi FH, Abdalrhim AD, Miller VM. Sex/gender medicine: The biological basis for personalized care in cardiovascular medicine. Circ J 2009; 73: 1774-1782.

4. Kambara H, Yamazaki T, Hayashi D, Kohro T, Okada Y, Nagai R; Japanese Coronary Artery Disease Study Investigators. Gender differences in patients with coronary artery disease in Japan: The Japanese Coronary Artery Disease Study (the JCAD study). Circ J 2009; 73: 912-917.

5. Masoudi FA, Havranek EP, Smith G, Fish RH, Steiner JF, Ordin DL, et al. Gender, age, and heart failure with preserved left ventricular systolic function. J Am Coll Cardiol 2003; 41: 217-223.

6. Klapholz M, Maurer M, Lowe AM, Messineo F, Meisner JS, Mitchell $\mathrm{J}$, et al. Hospitalization for heart failure in the presence of a normal left ventricular ejection fraction: Results of the New York Heart Failure Registry. J Am Coll Cardiol 2004; 43: 1432-1438.

7. Yancy CW, Lopatin M, Stevenson LW, De Marco T, Fonarow GC; ADHERE Scientific Advisory Committee and Investigators. Clinical presentation, management, and in-hospital outcomes of patients admitted with acute decompensated heart failure with preserved systolic function: A report from the Acute Decompensated Heart Failure National Registry (ADHERE) Database. J Am Coll Cardiol 2006; 47: $76-84$.

8. Frazier CG, Alexander KP, Newby LK, Anderson S, Iverson E, Packer M, et al. Associations of gender and etiology with outcomes in heart failure with systolic dysfunction: A pooled analysis of 5 randomized control trials. J Am Coll Cardiol 2007; 49: 1450-1458.

9. Hunt SA, Abraham WT, Chin MH, Feldman AM, Francis GS, Ganiats TG, et al. ACC/AHA 2005 Guideline Update for the Diagnosis and Management of Chronic Heart Failure in the Adult: A report of the American College of Cardiology/American Heart Association Task Force on Practice Guidelines (Writing Committee to Update the 2001 Guidelines for the Evaluation and Management of Heart Failure): Developed in collaboration with the American College of Chest Physicians and the International Society for Heart and Lung Transplantation: Endorsed by the Heart Rhythm Society. Circulation 2005; 112: e154-e235.

10. Munagala VK, Jacobsen SJ, Mahoney DW, Rodeheffer RJ, Bailey KR, Redfield MM. Association of newer diastolic function parameters with age in healthy subjects: A population-based study. J Am Soc Echocardiogr 2003; 16: 1049-1056.

11. Redfield MM, Jacobsen SJ, Borlaug BA, Rodeheffer RJ, Kass DA. Age- and gender-related ventricular-vascular stiffening: A community-based study. Circulation 2005; 112: 2254-2262.

12. Yamada H, Oki T, Mishiro Y, Tabata T, Abe M, Onose Y, et al. Effect of aging on diastolic left ventricular myocardial velocities measured by pulsed tissue Doppler imaging in healthy subjects. J Am Soc Echocardiogr 1999; 12: 574-581.

13. Onose Y, Oki T, Mishiro Y, Yamada H, Abe M, Manabe K, et al.
Influence of aging on systolic left ventricular wall motion velocities along the long and short axes in clinically normal patients determined by pulsed tissue doppler imaging. J Am Soc Echocardiogr 1999; 12: 921-926.

14. Daimon M, Watanabe H, Abe Y, Hirata K, Hozumi T, Ishii K, et al; the JAMP study investigators. Normal values of echocardiographic parameters in relation to age in a healthy Japanese population: The JAMP study. Circ J 2008; 72: 1859-1866.

15. Lang RM, Bierig M, Devereux RB, Flachskampf FA, Foster E, Pellikka PA, et al; Members of the Chamber Quantification Group. Recommendations for chamber quantification: A report from the American Society of Echocardiography's Guidelines and Standards Committee and the Chamber Quantification Writing Group, developed in conjunction with the European Association of Echocardiography, a branch of the European Society of Cardiology. J Am Soc Echocardiogr 2005; 18: 1440-1463.

16. Oh JK, Appleton CP, Hatle LK, Nishimura RA, Seward JB, Tajik AJ. The noninvasive assessment of left ventricular diastolic function with two-dimensional and Doppler echocardiography. J Am Soc Echocardiogr 1997; 10: 246-270.

17. Okura H, Takada Y, Yamabe A, Kubo T, Asawa K, Ozaki T, et al. Age- and gender-specific changes in the left ventricular relaxation: A Doppler echocardiographic study in healthy individuals. Circ Cardiovasc Imaging 2009; 2: 41-46.

18. Ling S, Dai A, Dilley RJ, Jones M, Simpson E, Komesaroff PA, et al. Endogenous estrogen deficiency reduces proliferation and enhances apoptosis-related death in vascular smooth muscle cells: Insights from the aromatase-knockout mouse. Circulation 2004; 109: 537 543.

19. Reis SE, Gloth ST, Blumenthal RS, Resar JR, Zacur HA, Gerstenblith G, et al. Ethinyl estradiol acutely attenuates abnormal coronary vasomotor responses to acetylcholine in postmenopausal women. Circulation 1994; 89: 52-60.

20. Billon-Galés A, Fontaine C, Douin-Echinard V, Delpy L, Berges H, Calippe B, et al. Endothelial estrogen receptor-alpha plays a crucial role in the atheroprotective action of 17beta-estradiol in lowdensity lipoprotein receptor-deficient mice. Circulation 2009; 120: 2567-2576.

21. Bursi F, Weston SA, Redfield MM, Jacobsen SJ, Pakhomov S, Nkomo $\mathrm{VT}$, et al. Systolic and diastolic heart failure in the community. JAMA 2006; 296: 2209-2216.

22. Owan TE, Hodge DO, Herges RM, Jacobsen SJ, Roger VL, Redfield MM. Trends in prevalence and outcome of heart failure with preserved ejection fraction. $N$ Engl J Med 2006; 355: 251-259.

23. Galderisi M. Diastolic dysfunction and diabetic cardiomyopathy: Evaluation by Doppler echocardiography. J Am Coll Cardiol 2006; 48: $1548-1551$.

24. From AM, Leibson CL, Bursi F, Redfield MM, Weston SA, Jacobsen SJ, et al. Diabetes in heart failure: Prevalence and impact on outcome in the population. Am J Med 2006; 119: 591-599. 